\title{
ljfis
}

Received: Jan. 3, 2021

Revised : Sep. 12, 2021

Accepted: Sep. 15, 2021

Correspondence to: Gautam Chandra Ray (gautomofcit@gmail.com)

${ }^{*}$ These authors contributed equally to this work. (CThe Korean Institute of Intelligent Systems

(c)This is an Open Access article distributed under the terms of the Creative Commons Attribution Non-Commercial License (http://creativecommons.org/licenses/by-nc/ 3.0/) which permits unrestricted noncommercial use, distribution, and reproduction in any medium, provided the original work is properly cited.

\section{Separation Axioms in Mixed Fuzzy Topological Spaces}

Gautam Chandra Ray and Hari Prasad Chetri

Department of Mathematics, Central Institute of Technology, Kokrajhar, Assam, India

\begin{abstract}
Herein, we define fuzzy $T_{0}$-space, fuzzy $T_{1}$-space, fuzzy $T_{2}$ (or Hausdorff), as well as fuzzy regular and fuzzy normal spaces in mixed fuzzy topological spaces, and then establish relationships among these spaces. We provide some results for the abovementioned spaces in mixed fuzzy topological spaces.
\end{abstract}

Keywords: Fuzzy topological spaces, Mixed fuzzy topology, Separation Axioms, $T_{0}$-space, $T_{1}$-space, $T_{2}$ (or Hausdorff) spaces

\section{Introduction}

The concept of mixing two topologies to obtain a new topology is not a new concept. In 1958, Alexiewicz and Semadeni [1] introduced and investigated a mixed topology on a set via norm spaces. Thereafter, many mathematicians worldwide investigated mixed topologies and discovered some interesting and applicable results regarding mixed topologies. In this regard, some remarkable results have been reported by Cooper [2], Buck [3], Wiweger [4], and many others. A new era began after the inception of the fuzzy set theory in 1965 by Zadeh [5]. Since the inception of the notion of fuzzy sets and fuzzy logic, fuzziness has been applied to studies in almost all branches of science and technology. The notion of fuzziness has been applied to topology for the first time by Chang [6], and fuzzy topological spaces have been introduced and then investigated extensively. The concept of strong separation and strong countability in fuzzy topological spaces was introduced and investigated by Stadler and de Prada Vicente [7]. In 1995, the concept of mixed fuzzy topological spaces was investigated from different perspectives by Das and Baishya [8]. Recently, in 2012, Tripathy and Ray [9] generalized the definition of mixed fuzzy topology introduced by Das and Baishya [8], by replacing fuzzy points with fuzzy sets. Tripathy and Ray [9] introduced the concept of countability base on a mixed fuzzy topology and proved the existence of three types of countability. However, this concept of mixed fuzzy topology is not a generalization of the concept of a crispy mixed topology (or simply mixed topology) by Wiweger [4], Alexiewicz and Semadeni [1], Cooper [2], and others. Ghanim et. al [10] investigated separation axioms. Recently, W. F. Al-Omeri [11] investigated a mixed b-fuzzy topology using the definition by Das and Baishya [8].

Most recently,Al-Shami and his colleagues [12-21] investigated separation axioms in topological spaces and obtained some important findings. Rashid and Ali [22] introduced separation axioms in a mixed fuzzy topology using the concept of mixed fuzzy topology defined by Das and Baishya [8]. Recently, Tripathy and Ray [9] generalized the definition of mixed fuzzy topology by Das and Baishya [8]. In this study, we redefined and investigated separation axioms in mixed 
fuzzy topological spaces introduced by Tripathy and Ray [9]. We investigated a few properties of separation axioms that varied slightly, as introduced by Rashid and Ali [22]. We have previously proven some results and constructed a few concrete numerical examples of $T_{0}, T_{1}, T_{2}$ (Hausdorff), and regular and normal mixed fuzzy topological spaces such that the spaces can be visualized more effectively. Herein, we provide the basic definitions in Section 2, although most of the concepts, definitions, and notations used herein are standard ones.

\section{Preliminaries}

Definition 1 [23]. Let $X$ be a non-empty set, and $I$ be the unit interval $[0,1]$. A fuzzy set $A$ in $X$ is characterized by a function $\mu_{A}: X \rightarrow I$, where $\mu_{A}$ is known as the membership function of $A . \mu_{A}(x)$ representing the membership grade of $x$ in $A$. The empty fuzzy set is defined as $\mu_{\phi}(x)=0$ for all $x \in X$. Additionally, $X$ can be regarded as a fuzzy set in itself, defined $\mu_{X}(t)=1$ for all $t \in X$. Furthermore, an ordinary subset $A$ of $X$ can be regarded as a fuzzy set in $X$ if its membership function is regarded as the usual characteristic function of $A$, i.e., $\mu_{A}(x)=1$, for all $x \in X$ and $\mu_{A}(x)=0$ for all $x \in X-A$. The two fuzzy sets $A$ and $B$ are equal if $\mu_{A}=\mu_{B}$. A fuzzy set $A$ is contained in a fuzzy set $B$, written as $A \subseteq B$, if $\mu_{A} \leq \mu_{B}$. The complement of a fuzzy set $A$ in $X$ is a fuzzy set $A$ in $X$, defined as $\mu_{A^{c}}=1-\mu_{A}$. We write $A^{c}=c o A$ to avoid confusion.

The union and intersection of a set $\left\{A_{i}: i \in I\right\}$ of fuzzy sets in $X$ to be written as $\cup A_{i}$ and $\cap A_{i}$, separately, are defined as follows:

$$
\mu_{\bigcup_{i \in I} A_{i}}(x)=\sup \left\{\mu_{A_{i}}(x): i \in I\right\} \text { for all } x \in X,
$$

and

$$
\mu_{\bigcap_{i \in I} A_{i}}(x)=\inf \left\{\mu_{A_{i}}(x): i \in I\right\} \text { for all } x \in X
$$

Definition $2[6]$. Let $I=[0,1], X$ be a non-empty set, and $I^{X}$ be a set of all mappings from $X$ to $I$, i.e., the class of all fuzzy sets in $X$.

A fuzzy topology on $X$ is a family $\tau$ of member $I^{X}$, such that

(i) $\overline{1}, \overline{0} \in \tau$.

(ii) For any finite subset $\mathfrak{B}=\left\{B_{i}\right\}_{i=1}^{n}$ of members of $\tau$, $\bigcap_{i=1}^{n}\left\{B_{i}\right\} \in \tau$.

(iii) For any arbitrary set $\Delta$ of members of $\tau, \bigcup_{B \in \Delta}\{B\} \in \tau$.
The pair $(X, \tau)$ is known as a fuzzy topological space (fts), and the members of $\tau$ are known as $\tau$-open fuzzy sets.

Definition 3 [24]. The closure of a fuzzy set $A$ in an fts $(X$, $\tau)$ is defined as the intersection of all closed supersets of $A$, i.e.,

$$
\bar{A}=\cap\{F: A \subset F, F \text { is a closed fuzzy set }\} .
$$

Definition 4 [23]. A fuzzy set in $X$ is known as a fuzzy point if and only if it assumes the value 0 for all $y \in X$ except one, e.g., $x \in X$. If its value at $x$ is $\alpha(0<\leq 1)$, then this point is denoted by $x$, and we refer to point $x$ as its support.

Definition 5 [23]. A fuzzy point $x_{\lambda}$ is contained in a fuzzy set $A$ or belongs to $A$, denoted by $x_{\alpha} \in A$, if and only if $\alpha \leq A(x)$.

Definition 6 [23]. Two fuzzy sets $A$ and $B$ in $X$ are intersecting if a point $x \in X$ exists such that $(A \cap B)(x) \neq 0$. In this case, $A$ and $B$ intersect at $x$.

Definition 7 [23]. A fuzzy set $A$ in a fts $(X, \tau)$ is known as the neighborhood of a fuzzy point $x_{\lambda} \in X$ if and only if $B \in \tau$ exists such that $x_{\lambda} \in B \subseteq A$; a neighborhood $A$ is known as an open neighborhood if and only if $A$ is fuzzy open. A family comprising all the neighborhoods of $x_{\lambda}$ is known as a system of neighborhoods of $x_{\lambda}$.

Definition $8[23]$. A fuzzy point $x_{\alpha}$ is quasi-coincident with a fuzzy set $A$, denoted by $x_{\alpha} q A$, if and only if $\alpha+A(x)>1$ or $\alpha>(A(x))^{c}$.

Definition 9 [23]. A fuzzy set $A$ is quasi-coincident with $B$ and is denoted by $A q B$ if and only if $x \in X$ exists such that $A(x)+B(x)>1$.

It is clear that if $A$ and $B$ are quasi-coincident at $x$, then both $A(x)$ and $B(x)$ are non-zero at $x$; hence, $A$ and $B$ intersect at $x$.

Definition 10 [23]. A fuzzy set $A$ in an fts $(X, \tau)$ is known as a quasi-neighborhood ( $Q$-neighborhood) of $x_{\lambda}$ if and only if $\exists A_{1} \in \tau$, such that $A_{1} \subseteq A$ and $x_{\lambda} q A_{1}$. A family $U_{x_{\lambda}}$ comprising all the $Q$-neighborhoods of $x$ is known as the $Q$-neighborhood system of $x$. The intersection of two $Q$ neighborhoods of $x_{\lambda}$ is a quasi-neighborhood.

Theorem 1 [8]. Let $\left(X, \tau_{1}\right)$ and $\left(X, \tau_{2}\right)$ be two fuzzy topological spaces. Consider fuzzy sets $\tau_{1}\left(\tau_{2}\right)=\left\{A \in I^{X}\right.$ : For any $x q A$, a $\tau_{2}$ - $Q$-neighborhood $A$ of $x$ exists such that $\tau_{1}$-closure $\left.\bar{A}_{\mid \alpha} \subseteq A\right\}$. Subsequently, this family of fuzzy sets will form a fuzzy topology on $X$, and this topology is known as a mixed fuzzy topology on $X$.

Definition $11[10]$. A fts $(X, \tau)$ is known as a fuzzy $T_{0^{-}}$ space if and only if for any pair of fuzzy points $x_{\alpha}$ and $y_{\beta}$ in 
$I^{X}$ with $x \neq y$, open fuzzy sets $U$ and $V$ exist in $\tau$ such that $x_{\alpha} \in U \subseteq \operatorname{co}\left(y_{\beta}\right)$ or $y_{\beta} \in V \subseteq \operatorname{co}\left(x_{\alpha}\right)$ in $\tau$.

Definition 12 [10]. A fts $(X, \tau)$ is known as a fuzzy $T_{1^{-}}$ space if and only if for any pair of fuzzy points $x_{\alpha}$ and $y_{\beta}$ in $I^{X}$ with $x \neq y$, open fuzzy sets $U$ and $V$ exist in $\tau$ such that $x_{\alpha} \in U$ and $y_{\beta} \notin U$, and $x_{\alpha} \notin V$ and $y_{\beta} \in V$.

Definition $13[10]$. A fts $(X, \tau)$ is known as the fuzzy Hausdorff or fuzzy $T_{2}$-space if and only if for any pair of fuzzy points $x_{\alpha}$ and $y_{\beta}$ in $I^{X}$ with $x \neq y$, open fuzzy sets $U$ and $V$ exist such that $x_{\alpha} \in U$ and $y_{\beta} \in V$, and $U \cap V=\overline{0}$.

Definition 14 [10]. A fts $(X, \tau)$ is known as fuzzy regular if and only if for any fuzzy point $x$ and a closed fuzzy set $B$ with $x \in B^{c}$,open fuzzy sets $U$ and $V$ exist in $\tau$ such that $x_{\alpha} \in U$ and $B \subseteq V$, and $U \subseteq 1-V$.

Proposition 1 [25].fts $(X, \tau)$ is known as a fuzzy regular space if and only if for any fuzzy point $x_{\alpha}$ in $I^{X}$ and $A \in \tau$ with $\alpha<A(x), B \in \tau$ exists such that $\alpha<B(x)$ and $\bar{B} \subseteq A$.

Definition $15[22]$. A fts $(X, \tau)$ is known as a fuzzy normal space if and only if for each closed set $B$ and an open fuzzy set $U$ in $(X, \tau)$ where $B \subseteq U, V \in \tau$ exists such that $B \subseteq V \subseteq$ $\bar{V} \subseteq U$.

Lemma $1[8]$. Let $\tau_{1}$ and $\tau_{2}$ be two fuzzy topological spaces on set $X$. If every $\tau_{1}$-Q-neighbourhood of $x$ is a $\tau_{2^{-}}$ $Q$-neighbourhood of $x$ for all fuzzy points $x$, then $\tau_{1}$ is coarser than $\tau_{2}$.

Theorem $2[9]$. Let $\left(X, \tau_{1}\right)$ and $\left(X, \tau_{2}\right)$ be two fuzzy topological spaces. Consider fuzzy sets $\tau_{1}\left(\tau_{2}\right)=\left\{A \in I^{X}\right.$ : For any fuzzy set $B$ in $X$ with $A q B$, a $\tau_{2}$-open set $A_{1}$ exists such that $A_{1} q B$ and $\tau_{1}$-closure $\left.\bar{A}_{1} \subseteq A\right\}$. Subsequently, this family of fuzzy sets will form a fuzzy topology on $X$, and this topology is known as a mixed fuzzy topology on $X$.

Theorem 3 [8]. Let $\tau_{1}$ and $\tau_{2}$ be two fuzzy topological spaces on set $X$. Subsequently, the mixed fuzzy topology $\tau_{1}\left(\tau_{2}\right)$ is coarser than $\tau_{2}$. This is represented as $\tau_{1}\left(\tau_{2}\right) \subseteq \tau_{2}$.

Proof. Because every fuzzy point is also a fuzzy singleton set, the theorem is valid with respect to the definition in Theorem 2.

\section{Results}

In this section, we analogously define the basic definition of $T_{0}, T_{1}, T_{2}$ (or Hausdorff), as well as regular and normal mixed fuzzy topological spaces $\left(X, \tau_{1}\left(\tau_{2}\right)\right)$, and then provide some results in mixed fuzzy topological spaces. The definitions used herein are as follows:

Definition 16. A mixed fts $\left(X, \tau_{1}\left(\tau_{2}\right)\right)$ is known as a fuzzy
$T_{0}$-space if and only if for any pair of fuzzy points $x_{\alpha}$ and $y_{\beta}$ in $I^{X}$ with $x \neq y$, open fuzzy sets $U$ and $V$ exist in $\tau_{1}\left(\tau_{2}\right)$ such that $x_{\alpha} \in U \subseteq \operatorname{co}\left(y_{\beta}\right)$ or $y_{\beta} \in V \subseteq \operatorname{co}\left(x_{\alpha}\right)$ in $\tau_{1}\left(\tau_{2}\right)$.

Definition 17. A mixed fts $\left(X, \tau_{1}\left(\tau_{2}\right)\right)$ is known as a mixed fuzzy $T_{1}$-space if and only if for any pair of fuzzy points $x_{\alpha}$ and $y_{\beta}$ in $I^{X}$ with $x \neq y$, open fuzzy sets $U$ and $V$ in $\tau_{1}\left(\tau_{2}\right)$ exist such that $x_{\alpha} \in U$ and $y_{\beta} \notin U$ as well as $x_{\alpha} \notin V$ and $y_{\beta} \in V$.

Definition 18. A mixed fts $\left(X, \tau_{1}\left(\tau_{2}\right)\right)$ is known as a fuzzy Hausdorff or fuzzy $T_{2}$-space if and only if for any pair of fuzzy points $x_{\alpha}$ and $y_{\beta}$ in $I^{X}$ with $x \neq y$,open fuzzy sets $U$ and $V$ exist in $\tau_{1}\left(\tau_{2}\right)$ such that $x_{\alpha} \in U$ and $y_{\beta} \in V$, and $U \cap V=\overline{0}$.

Definition 19. A mixed fts $\left(X, \tau_{1}\left(\tau_{2}\right)\right)$ is known as a fuzzy regular space if and only if for any fuzzy point $x_{\alpha}$ in $I^{X}$ and a closed set $B$ in $\tau_{1}\left(\tau_{2}\right)$ with $x \in B^{c}$, open fuzzy sets $U$ and $V$ exist in $\tau_{1}\left(\tau_{2}\right)$ such that $x_{\alpha} \in U$ and $B \subseteq V$, and $U \subseteq 1-V$.

Definition 20. A mixed fts $\left(X, \tau_{1}\left(\tau_{2}\right)\right)$ is known as a fuzzy normal space if and only if for each closed fuzzy set $B$ and an open fuzzy set $U$ in $\tau_{1}\left(\tau_{2}\right)$ where $B \subseteq U, V \in \tau_{1}\left(\tau_{2}\right)$ exists such that $B \subseteq V \subseteq \bar{V} \subseteq U$.

Example1. Let us consider the following example of mixed fuzzy topology, which comprises fuzz $T_{0}$, fuzzy $T_{1}$ and fuzzy $T_{2}$.

Let $X=\{x, y\}$ and $x \neq y$; therefore

$$
\begin{aligned}
\tau_{1}= & \{\overline{0}, \overline{1},\{(x, \alpha),(y, 0)\},\{(x, 0),(y, \alpha)\}, \\
& \{(x, \alpha),(y, \beta)\} \mid \alpha, \beta \in[0.6,1]\},
\end{aligned}
$$

and

$$
\begin{aligned}
\tau_{2}= & \{\overline{0}, \overline{1},\{(x, 0.3),(y, 0)\},\{(x, 0),(y, 0.3)\}, \\
& \{(x, 0.5),(y, 0)\},\{(x, 0),(y, 0.5)\}, \\
& \{(x, 0.3),(y, 0.3)\},\{(x, 0.5),(y, 0.5)\}, \\
& \{(x, 1),(y, 0)\},\{(x, 0),(y, 1)\}\}
\end{aligned}
$$

are fuzzy Hausdorff topological spaces. We constructed $\tau_{1}\left(\tau_{2}\right)$ from these topologies. In addition, $\tau_{1}\left(\tau_{2}\right) \subseteq \tau_{2}$. Therefore we verified the belongingness of $\{(x, 0.3),(y, 0.3)\},\{(x, 0.5)$, $(y, 0.5)\},\{(x, 0.3),(y, 0)\},\{(x, 0),(y, 0.3)\},\{(x, 0.5),(y$, $0)\},\{(x, 0),(y, 0.5)\},\{(x, 1),(y, 0)\}$, and $\{(x, 0),(y, 1)\}$ in $\tau_{1}\left(\tau_{2}\right)$. As an example, $=\{(x, 0.3),(y, 0.3)\} \in \tau_{2}$ for $A_{1}=\{(x, \delta): \delta>0.7\} ; A q A_{1}$ and the $\tau_{1}$ closure of $(A=\{(x$, $0.3),(y, 0.3)\})=A \subseteq A$. Because $\{(x, 0.7),(y, 0.7)\}$ is an open set in $\tau_{1},\{(x, 0.3),(y, 0.3)\}$ is a closed set in $\tau_{1}$. (Similarly, for $A_{2}=\{(y, \delta): \delta>0.7\}$ or $A_{3}=\{(x, \gamma),(y$, $\delta) \mid \gamma, \delta \in(0.7,1]\} ; A q A_{2}$ and $A q A_{3}$ are also a $\tau_{1}$ closure of 
$(A=\{(x, 0.3),(y, 0.3)\})=A \subseteq A$.

Therefore $A \in \tau_{1}\left(\tau_{2}\right)$. However, for $B=\{(x, 0.5),(y$, $0.5)\} \in \tau_{2}$ and $A_{2}=\{(x, \delta): \delta>0.5\} B q A_{2}$ and the $\tau_{1}$ closure of $(B=\{(x, 0.5),(y, 0.5)\})=\overline{1} \not \subset B$.

Therefore, $B \notin \tau_{1}\left(\tau_{2}\right)$. Similarly, as in the case of $A=\{(x$, $0.3),(y, 0.3)\} \in \tau_{2},\{(x, 1),(y, 0)\}$ and $\{(x, 0),(y, 1)\}$ will be members of $\tau_{1}\left(\tau_{2}\right)$. Furthermore, $\tau_{1}$ is the closure of $\{(x$, $1),(y, 0)\}=\{(x, 1),(y, 0)\}$, as $\{(x, 0),(y, 1)\}$ is an open set in $\tau_{1}$; therefore, the complement $\{(x, 1),(y, 0)\}$ is closed. In addition, the $\tau_{1}$ closure of $\{(x, 0),(y, 1)\}=\{(x, 0),(y, 1)\}$ as $\{(x, 1),(y, 0)\}$ is an open set in $\tau_{1}$.

The complement of $\{(x, 0.3),(y, 0)\}$ in $\tau_{1}$ is $\{(x, 0.7)$, $(y, 1)\}$, which is an open set such that $\{(x, 0.3),(y, 0)\}$ is a closed set in $\tau_{1}$. Therefore, as in the case of $A=\{(x, 0.3)$, (y, $0)\} \in \tau_{1}\left(\tau_{2}\right)$, we have $\{(x, 0),(y, 0.3)\} \in \tau_{1}\left(\tau_{2}\right)$.

However, the complement of $\{(x, 0.5),(y, 0)\}$ in $\tau_{1}$ is $\{(x$, $0),(y, 0.5)\}$ and not fuzzy open in $\tau_{1}$; therefore, $\{(x, 0.5)$, $(y, 0)\}$ is not fuzzy closed, and the $\tau_{1}$-closure of $\{(x, 0.5),(y$, $0)\} \not \subset\{(x, 0.5),(y, 0)\}$.

Similarly, $\{(x, 0.5),(y, 0)\} \notin \tau_{1}\left(\tau_{2}\right)$ and $\{(x, 0),(y, 0.5)\} \notin$ $\tau_{1}\left(\tau_{2}\right)$

Therefore, $\tau_{1}\left(\tau_{2}\right)=\{\overline{0}, \overline{1},\{(x, 0.3),(y, 0.3)\},\{(x, 0.3),(y$, $0)\},\{(x, 0),(y, 0.3)\},\{(x 1),(y, 0)\},\{(x, 0),(y, 1)\}\}$.

Next, we prove that $\tau_{1}\left(\tau_{2}\right)$ is fuzzy $T_{0}$, fuzzy- $T_{1}$, and fuzzy$T_{2}$.

For $x_{\alpha}$ and $y_{\beta}$ in $I^{X}, x_{\alpha} \in\{(x, 1),(y, 0)\} \subseteq \operatorname{co}\left(y_{\beta}\right)$ and $y_{\beta} \in\{(x, 0),(y, 1)\} \subseteq \operatorname{co}\left(x_{\alpha}\right)$.

Additionally, $\{(x, 1),(y, 0)\} \cap\{(x, 0),(y, 1)\}=\overline{0}$.

Therefore, $\tau_{1}\left(\tau_{2}\right)$ qualifies as fuzzy- $T_{0}$, fuzzy- $T_{1}$, and fuzzy$T_{2}$.

Example 2. Let $X=\{x, y\}$; subsequently, $\tau_{1}=\{\overline{1}, \overline{0},\{(x$, $0),(y, 1)\}\}$ and $\tau_{2}=\{\overline{1}, \overline{0},\{(x, 1),(y, 0)\},\{(x, 0.7),(y$, $0.7)\},\{(x, 0.7),(y, 0)\},\{(x, 1),(y, 0.7)\}\}$ are fuzzy topologies. Therefore, $\tau_{1}\left(\tau_{2}\right)=\{\overline{1}, \overline{0},\{(x, 1),(y, 0)\}\}$, since $\{(x$, $1),(y, 0)\}$ is a closed fuzzy set in $\tau_{1}$. For any fuzzy set $B$ in $X$ with $\{(x, 1),(y, 0)\} q B$, we have a $\tau_{2}$-open fuzzy set $\{(x, 1),(y, 0)\}$ such that $\{(x, 1),(y, 0)\} q B$ and $\tau_{1}$-closure $(\overline{\{(x, 1),(y, 0)\}}) \subseteq\{(x, 1),(y, 0)\}$.

Clearly, $\tau_{1}\left(\tau_{2}\right)$ is mixed fuzzy $T_{0}$ but not mixed fuzzy $T_{1}$, as $x_{\alpha} \in\{(x, 1),(y, 0)\} \subseteq \operatorname{co}\left(y_{\beta}\right)$ and $\nexists A \in \tau_{1}\left(\tau_{2}\right)$ such that $y_{\alpha} \in A \subseteq \operatorname{co}\left(x_{\beta}\right)$.

Next, we consider a mixed fuzzy topological space that is fuzzy $T_{1}$ but not fuzzy $T_{2}$.

Example 3. Let $X=\{x, y, z\}$; subsequently, $\tau_{1}=\{\overline{1}, \overline{0}$, $\{(x, 1),(y, 1)(z, 0)\},\{(x, 1),(y, 0)(z, 1)\},\{(x, 0),(y, 1)(z$, $1)\},\{(x, 1),(y, 0)(z, 0)\},\{(x, 0),(y, 1)(z, 0)\},\{(x, 0),(y$, $0)(z, 1)\},\{(x, 0.7),(y, 0)(z, 0)\},\{(x, 0.7),(y, 1)(z, 0)\},\{(x$, $0.7),(y, 0)(z, 1)\},\{(x, 0.7),(y, 1)(z, 1)\}\}$ and $\tau_{2}=\{\overline{1}, \overline{0}$, $\{(x, 1),(y, 1)(z, 0)\},\{(x, 1),(y, 0)(z, 1)\},\{(x, 0),(y, 1)(z$, $1)\},\{(x, 1)(y, 0)(z, 0)\},\{(x, 0),(y, 1)(z, 0)\},\{(x, 0),(y$, $0)(z, 1)\}\}$ are fuzzy topologies.

Therefore, $\tau_{1}\left(\tau_{2}\right)=\{\overline{1}, \overline{0},\{(x, 1),(y, 1)(z, 0)\},\{(x, 1),(y$, $0)(z, 1)\},\{(x, 0),(y, 1)(z, 1)\},\{(x, 1),(y, 0)(z, 0)\},\{(x, 0)$, $(y, 1)(z, 0)\},\{(x, 0),(y, 0)(z, 1)\}\}$.

Because every open fuzzy set in $\tau_{2}$ is a closed fuzzy set in $\tau_{1}$. Therefore, every open fuzzy set in $\tau_{2}$ is an open fuzzy set in $\tau_{1}\left(\tau_{2}\right)$, as shown in Example 2.

Next, we demonstrate that for every pair $x_{\alpha}, y_{\beta}, y_{\alpha}, z_{\beta}$, or $z_{\alpha}, x_{\beta}$,open fuzzy sets $U$ and $V$ exist in $\tau_{1}\left(\tau_{2}\right)$ such that $x_{\alpha} \in U \subseteq \operatorname{co}\left(y_{\beta}\right)$ and $y_{\beta} \in V \subseteq \operatorname{co}\left(x_{\alpha}\right)$. We will verify this only for the pair $x_{\alpha}, y_{\beta}$; similarly, other cases can be completed. We have open fuzzy sets $\{(x, 1),(y, 0)(z, 1)\}$ and $\{(x, 0),(y, 1)(z, 1)\}$ in $\tau_{1}\left(\tau_{2}\right)$ such that $x_{\alpha} \in\{(x, 1),(y, 0)(z$, $1)\} \subseteq c o\left(y_{\beta}\right)$ and $y_{\beta} \in\{(x, 0),(y, 1)(z, 1)\} \subseteq c o\left(x_{\alpha}\right)$.

Therefore, $\tau_{1}\left(\tau_{2}\right)$ is fuzzy $T_{1}$.

However, $\{(x, 1),(y, 0)(z, 1)\} \not \subset c o(\{(x, 0),(y, 1)(z, 1)\})$. Therefore, $\tau_{1}\left(\tau_{2}\right)$ is not a mixed fuzzy $T_{2}$ space.

Next, we establish the following theorems:

Theorem 4. If $\tau_{1} \subseteq \tau_{2}$ and $\tau_{1}$ is fuzzy regular, then $\tau_{1} \subseteq$ $\tau_{1}\left(\tau_{2}\right)$.

Proof. Let $A$ be a $\tau_{1}$ - $Q$-neighborhood of $x_{\alpha}$, then $\exists A_{1} \in \tau_{1}$ such that

$$
\begin{aligned}
& x_{\alpha} q A_{1} \subseteq A \\
& \Rightarrow \alpha+A_{1}(x)>1 \quad(0<\alpha \leq 1) \\
& \Rightarrow A_{1}(x)>1-\alpha=\beta \text { (say) } \\
& \Rightarrow A_{1}(x)>\beta, \text { where } 0<\beta \leq 1 \\
& \Rightarrow B \in \tau_{1} \text { exists such that } \beta<B(x) \text { and } \tau_{1} \text {-closure of } \\
& B \subseteq A_{1}
\end{aligned}
$$

(Since $\tau_{1}$ is fuzzy regular).

Therefore,

$$
\begin{aligned}
& 1-\alpha<B(x) \\
& \Rightarrow 1<B(x)+\alpha \text { and } \tau_{1} \text {-closure } \bar{B} \subseteq A_{1} \\
& \Rightarrow x_{\alpha} q B, \text { and } B \in \tau_{2} \text { and } \tau_{1} \text {-closure } \bar{B} \subseteq A_{1} \subseteq A \\
& \left.\Rightarrow A \in \tau_{1}\left(\tau_{2}\right) \text { (By definition of } \tau_{1}\left(\tau_{2}\right)\right) \\
& \Rightarrow A \text { is a } \tau_{1}\left(\tau_{2}\right) \text { quasi-neighborhood of } x_{\alpha} \\
& \Rightarrow \tau_{1} \subseteq \tau_{1}\left(\tau_{2}\right) .
\end{aligned}
$$


Hence, the proof is completed.

Theorem 5. If $\tau_{1} \subseteq \tau_{2}$ and $\tau_{1}$ are fuzzy regular, then $\tau_{1}\left(\tau_{2}\right)$ is a fuzzy- $T_{0}$, fuzzy- $T_{1}$, and fuzzy- $T_{2}$ (or Hausdorff) space.

Proof. If $\tau_{1}$ is fuzzy regular, then $\tau_{1}$ is a fuzzy- $T_{0}$, fuzzy- $T_{1}$, and fuzzy- $T_{2}$ (or Hausdorff) space.

Furthermore, $\tau_{1} \subseteq \tau_{1}\left(\tau_{2}\right)$ (by Theorem 4).

1) We prove that $\tau_{1}\left(\tau_{2}\right)$ is a fuzzy $T_{0}$-space.

Let $x_{\alpha}, y_{\beta}$ be any two fuzzy points such that $x \neq y$. Because $\tau_{1}$ is a fuzzy $T_{0}$-space, without loss of generality, let an open fuzzy set $U \in \tau_{1}$ such that

$$
\begin{aligned}
& x_{\alpha} \in U \subseteq \operatorname{co}\left(y_{\beta}\right) \\
& \Rightarrow x_{\alpha} \in U \in \tau_{1}\left(\tau_{2}\right) \text { and } y_{\beta} \notin U\left[\text { since } \tau_{1} \subseteq \tau_{1}\left(\tau_{2}\right)\right] \\
& \Rightarrow \exists \text { open fuzzy set } U \in \tau_{1}\left(\tau_{2}\right) \text { such that } x_{\alpha} \in U \subseteq \operatorname{co}\left(y_{\beta}\right) \\
& \Rightarrow \tau_{1}\left(\tau_{2}\right) \text { is fuzzy } T_{0} \text { space. }
\end{aligned}
$$

2) Next, we prove that $\tau_{1}\left(\tau_{2}\right)$ is a fuzzy $T_{1}$-space.

Let $x_{\alpha}$ and $y_{\beta}$ be any two fuzzy points such that $x \neq y$. Because $\tau_{1}$ is a fuzzy $T_{1}$-space, open fuzzy sets $V_{1}$ and $V_{2}$ in $\tau_{1}$ exist such that $x_{\alpha} \in V_{1}$ and $y_{\beta} \notin V_{1}$, and $x_{\alpha} \notin V_{2}$ and $y_{\beta} \in V_{2}$. Since $\tau_{1} \subseteq \tau_{1}\left(\tau_{2}\right)$, therefore $V_{1}$ and $V_{2}$ are in $\tau_{1}\left(\tau_{2}\right)$ such that $x_{\alpha} \in V_{1}$ and $y_{\beta} \notin V_{1}$, and $x_{\alpha} \notin V_{2}$ and $y_{\beta} \in V_{2}$. Therefore, $\tau_{1}\left(\tau_{2}\right)$ is a fuzzy $T_{1}$-pace.

3) Finally, we prove that $\tau_{1}\left(\tau_{2}\right)$ is a fuzzy Hausdorff space.

Let $x_{\alpha}$ and $y_{\beta}$ be any two fuzzy points such that $x \neq y$.

$\Rightarrow V_{1}$ and $V_{2}$ exist in $\tau_{1}$ such that $x_{\alpha} \in V_{1}$ and $y_{\beta} \in V_{2}$ and $V_{1} \cap V_{2}=\overline{0}$ (since $\tau_{1}$ is fuzzy Hausdorff).

$\Rightarrow V_{1}$ and $V_{2}$ are in $\tau_{1}\left(\tau_{2}\right)$ such that $x_{\alpha} \in V_{1}$ and $y_{\beta} \in V_{2}$, and $V_{1} \cap V_{2}=\overline{0}$. [since $\tau_{1} \subseteq \tau_{1}\left(\tau_{2}\right)$ ]

$\Rightarrow \tau_{1}\left(\tau_{2}\right)$ is a fuzzy Hausdorff space.

Theorem 6. If $\tau_{1}\left(\tau_{2}\right)$ is a fuzzy $T_{0}$-space, then $\tau_{2}$ is also a fuzzy $T_{0}$-space.

Proof. We assume $\tau_{1}\left(\tau_{2}\right)$ to be a fuzzy $T_{0}$-space.

Let $x_{\alpha}$ and $y_{\beta}$ be any two fuzzy points such that $x \neq y$.

Without loss of generality, let an open fuzzy set $U \in \tau_{1}\left(\tau_{2}\right)$ exist such that $x_{\alpha} \in U \subseteq c o\left(y_{\beta}\right)$.

Since $\tau_{1}\left(\tau_{2}\right) \subseteq \tau_{2}, U \in \tau_{2}$ such that $x_{\alpha} \in U \subseteq c o\left(y_{\beta}\right)$.

Hence, $\tau_{2}$ is a fuzzy $T_{0}$-space.

Theorem 7. If $\tau_{1}\left(\tau_{2}\right)$ is a fuzzy $T_{1}$-space, then $\tau_{2}$ is also a fuzzy $T_{1}$-space.

Proof. Let us assume that $\tau_{1}\left(\tau_{2}\right)$ is a fuzzy $T_{1}$-space.

Let $x_{\alpha}$ and $y_{\beta}$ be any two fuzzy points such that $x \neq y$. Because $\tau_{1}\left(\tau_{2}\right)$ is a fuzzy $T_{1}$-space, open fuzzy sets $V_{1}$ and $V_{2}$ exist in $\tau_{1}\left(\tau_{2}\right)$ such that $x_{\alpha} \in V_{1}$ and $y_{\beta} \notin V_{1}, x_{\alpha} \notin V_{2}$, and $y_{\beta} \in V_{2}$. Since $\tau_{1}\left(\tau_{2}\right) \subseteq \tau_{2}, V_{1}$ and $V_{2}$ exist in $\tau_{2}$ such that $x_{\alpha} \in V_{1}$ and $y_{\beta} \notin V_{1}$, and $x_{\alpha} \notin V_{2}$ and $y_{\beta} \notin V_{2}$.
Hence, $\tau_{2}$ is also a fuzzy $T_{1}$-space.

Theorem 8. If $\tau_{1}\left(\tau_{2}\right)$ is a fuzzy $T_{2}$-space (or Hausdorff), then $\tau_{2}$ is also a fuzzy $T_{2}$-space.

Proof. Let $x_{\alpha}$ and $y_{\beta}$ be any two fuzzy points such that $x \neq y$.

Because $\tau_{1}\left(\tau_{2}\right)$ is fuzzy Hausdorff, $V_{1}$ and $V_{2}$ exist in $\tau_{1}\left(\tau_{2}\right)$ such that $x_{\alpha} \in V_{1}$ and $y_{\beta} \in V_{2}$, and $V_{1} \cap V_{2}=\overline{0}$.

Hence, $V_{1}$ and $V_{2}$ exist in $\tau_{2}$ such thauto-at let $x_{\alpha} \in V_{1}$ and $y_{\beta} \in V_{2}$, and $V_{1} \cap V_{2}=\overline{0}$ (since $\left.\tau_{1}\left(\tau_{2}\right) \subseteq \tau_{2}\right)$.

Hence, $\tau_{2}$ is also a fuzzy $T_{2}$-space.

Theorem 9. Let $\tau_{1} \supseteq \tau_{2}$ and $\tau_{2}$ be fuzzy regular; therefore, $\tau_{1}\left(\tau_{2}\right)$ is also fuzzy regular.

Proof. Let $x_{\alpha}$ be a fuzzy point and $C$ be a closed fuzzy set in $\tau_{1}\left(\tau_{2}\right)$ such that $x_{\alpha} \in C^{c}$.

Therefore, $C$ is a closed fuzzy set in $\tau_{1}\left(\tau_{2}\right)$.

$\Rightarrow 1-C$ is an open fuzzy set in $\tau_{1}\left(\tau_{2}\right)$.

$\Rightarrow 1-C$ is an open fuzzy set in $\tau_{2}$ (since $\left.\tau_{1}\left(\tau_{2}\right) \subseteq \tau_{2}\right)$.

$\Rightarrow C$ is closed in $\tau_{2}$.

$\Rightarrow$ open fuzzy sets $A_{1}$ and $A_{2}$ exist in $\tau_{2}$ such that $x_{\alpha} \in A_{1}$ and $C \subseteq A_{2}$ and $A_{1} \subseteq 1-A_{2}$.

Next, for any fuzzy set $B$ such that $B q A_{1}$

$\Rightarrow B(z)+A_{1}(z)>1$, for some $z \in X$

$\Rightarrow A_{1}(z)>1-B(z)$

$\Rightarrow A_{1}(z)>r$, where $r=1-B(z)$.

The fuzzy regularity of $\tau_{2}$ guarantees that $\exists A_{2} \in \tau_{2}$ such that $A_{2}(z)>r$ and $A_{2} \subseteq \tau_{2}-\operatorname{cl}\left(A_{t} \subseteq A_{1}\right.$, since $\tau_{1} \supseteq \tau_{2}$.

Therefore,

$\tau_{1}-\operatorname{cl}\left(A_{2}\right)=\cap\left\{B \mid A_{2} \subseteq B\right.$, where $B$ is a closed fuzzy set in $\left.\tau_{1}\right\}$

$\subseteq \cap\left\{B \mid A_{2} \subseteq B, B\right.$ is a closed fuzzy set in $\left.\tau_{2}\right\}$ $=\tau_{2}-\operatorname{cl}\left(A_{2}\right)$. [Every closed fuzzy set in $\tau_{2}$ is a closed fuzzy set in $\tau_{1}$ ].

Therefore, $A_{2} q B$ and $A_{2} \subseteq \tau_{1}-\operatorname{cl}\left(A_{2}\right) \subseteq A_{1}$.

Furthermore, $A_{1} \in \tau_{1}\left(\tau_{2}\right)$, and similarly, we can show that $A_{2} \in \tau_{1}\left(\tau_{2}\right)$.

$\Rightarrow$ open fuzzy sets $A_{1}$ and $A_{2}$ exist in $\tau_{1}\left(\tau_{2}\right)$ such that $x_{\alpha} \in$ $A_{1}$ and $C \subseteq A_{2}$, and $A_{1} \subseteq 1-A_{2}$.

Hence, $\left(X, \tau_{1}\left(\tau_{2}\right)\right)$ is a fuzzy regular space.

Corollary 1. For any non-empty crisp set $X$, if $\tau_{2} \subseteq \tau_{1}\left(\tau_{2}\right)$, then $\tau_{2}=\tau_{1}\left(\tau_{2}\right)$ because $\tau_{1}\left(\tau_{2}\right) \subseteq \tau_{2}$.

Next, we consider a mixed fuzzy topology comprising fuzzy regular and fuzzy normal. 
Example 4. Let $X=\{x, y\}$ and $x \neq y$. Let $\tau_{1}$ be the fuzzy topology generated by the base $\mathfrak{B}_{1}=\left\{x_{\alpha} \mid \alpha \in(0\right.$, 1] $\} \cup\left\{y_{\beta} \mid \beta \in(0,1]\right\} \cup\{\emptyset\}$ and $\tau_{2}$ be the fuzzy topology generated by the base $\mathfrak{B}_{2}=\left\{x_{\alpha} \mid \alpha \in(0,1]\right.$, where $\alpha$ is a rational number $\} \cup\left\{y_{\beta} \mid \beta \in(0,1]\right.$ and $\beta$ is a rational number $\} \cup\{\emptyset\}$. Therefore, $\tau_{1}$ and $\tau_{2}$ are fuzzy regular and fuzzy normal spaces, respectively. Next, we show that the mixed fuzzy topology $\tau_{1}\left(\tau_{2}\right)$ is the same as the fuzzy topology $\tau_{2}$. We know that $\tau_{1}\left(\tau_{2}\right) \subseteq \tau_{2}$. Moreover, for any element in the base, e.g., $B=\left\{x_{\alpha} \mid \alpha \in(0,1]\right.$, where $\alpha$ is a rational number $\}$, the fuzzy set $C=\{(x, 1-\alpha),(y, 1)\}$ is an open fuzzy set in $\tau_{1}$ and a complement of $B$ in $\tau_{1}$. Therefore $\mathrm{B}$ is a closed fuzzy set in $\tau_{1}$, and $\tau_{1}$-closure of $B=B$. Therefore, for any fuzzy set $A$ with $A q B \exists \tau_{2}$ open fuzzy set $B$ such that $A q B$ and $\tau_{1}$-closure of $B=B$. Therefore, $B \in \tau_{1}\left(\tau_{2}\right)$. Similarly, any base element $\left\{y_{\beta} \mid \beta \in(0,1]\right.$, where $\beta$ is a rational number $\}$ is also in $\tau_{1}\left(\tau_{2}\right)$; this implies $\tau_{2} \subseteq \tau_{1}\left(\tau_{2}\right)$. Therefore, $\tau_{1}\left(\tau_{2}\right)=\tau_{2}$.

Next, we prove the fuzzy normality and fuzzy regularity of $\tau_{1}\left(\tau_{2}\right)$.

For the fuzzy regularity, let $x$ be any fuzzy point and $B$ be a closed fuzzy set in $\tau_{1}\left(\tau_{2}\right)$ such that

$$
\begin{aligned}
& x \in B^{c} \\
& \Rightarrow B^{c}(x) \geq \lambda \\
& \Rightarrow 1-B(x) \geq \lambda \\
& \Rightarrow B(x) \leq 1-\lambda \\
& \Rightarrow B(x)=1-\lambda \text { or } B(x)<1-\lambda .
\end{aligned}
$$

If $B(x)=1-\lambda$, then $1-\lambda$ is a rational number.

$B \subseteq\{(x, 1-\lambda),(y, 1)\}=V$ is an open fuzzy set in $\tau_{1}\left(\tau_{2}\right)$ and $x \in U=\{(x, \lambda),(y, 0)\} \in \tau_{1}\left(\tau_{2}\right)$ such that $U=\{(x, \lambda)$, $(y, 0)\} \subseteq 1-V=\{(x, \lambda),(y, 0)\}$. Therefore, in this case, $\tau_{1}\left(\tau_{2}\right)$ is fuzzy regular.

Next, if $B(x)<1-$, then $\exists$ is a rational number $\delta$ such that $B(x)<\delta<1-\lambda$.

Therefore, $B \subseteq\{(x, \delta),(y, 1)\}=V$ is an open fuzzy set in $\tau_{1}\left(\tau_{2}\right)$.

Furthermore,

$$
\begin{aligned}
& \delta<1-\lambda \\
& \Rightarrow 1-\delta>1-(1-\lambda) \\
& \Rightarrow 1-\delta>\lambda
\end{aligned}
$$

$\exists$ is a rational number $r$ such that $1-\delta>r>\lambda$
$\Rightarrow\{(x, r),(y, 0)\}=U$ is an open set in $\tau_{1}\left(\tau_{2}\right)$ such that $x \in U$ and $V^{c}=\{(x, 1-\delta),(y, 0)\}$, resulting in $U \subseteq V^{c}$; therefore, $\tau_{1}\left(\tau_{2}\right)$ is fuzzy regular in both cases.

Next, we prove that $\tau_{1}\left(\tau_{2}\right)$ is fuzzy normal.

Let $B$ be any closed fuzzy set and $U$ be an open fuzzy set such that $B \subseteq U$.

$\Rightarrow B(x) \leq U(x), \forall x \in X$ and $U$ is an open fuzzy set in $\tau_{1}\left(\tau_{2}\right) . B(x)=1-r$ for some rational number $r \in(0,1]$

$\Rightarrow B(x) \leq \frac{B(x)+U(x)}{2} \leq U(x)$ and $\frac{B(x)+U(x)}{2}$ is a rational number in $(0,1]$.

Therefore, $V(x)=\frac{B(x)+U(x)}{2}$ and $V(y)=\frac{B(y)+U(y)}{2}$ defines a fuzzy open set in $\tau_{1}\left(\tau_{2}\right)$.

Furthermore, $B(x) \leq V(x) \leq U(x) \forall x \in X$. Similarly, $1-V$ yields rational values for both $x$ and $y$; therefore, $1-V$ is open in $\tau_{1}\left(\tau_{2}\right) \Rightarrow V$ is fuzzy closed as well as $\Rightarrow V(x)=\bar{V}(x)$ $\forall x \in X$.

$$
\begin{aligned}
& \Rightarrow B(x) \leq V(x) \leq \bar{V}(x) \leq U(x) \forall x \in X \\
& \Rightarrow B(x) \leq V(x) \leq \bar{V}(x) \leq U(x) .
\end{aligned}
$$

Therefore, $\tau_{1}\left(\tau_{2}\right)$ is a normal mixed fuzzy topological space.

Theorem 10. If $\tau_{1} \supseteq \tau_{2}$ and $\tau_{2}$ are fuzzy normal, then $\tau_{1}\left(\tau_{2}\right)$ is also fuzzy normal.

Proof. Since $\tau_{2}$ is fuzzy normal, it is fuzzy regular. Therefore, from Corollary 1 , we obtain $\tau_{1}\left(\tau_{2}\right)=\tau_{2}$. Hence, $\tau_{1}\left(\tau_{2}\right)$ is also normal.

Theorem 11. A fuzzy $T_{2}$ mixed fts $\left(X, \tau_{1}\left(\tau_{2}\right)\right)$ is a mixed fuzzy $T_{1}$-space, and a mixed fuzzy $T_{1}$ space is a mixed fuzzy $T_{0}$-space.

Proof. Let $\left(X, \tau_{1}\left(\tau_{2}\right)\right)$ be a $T_{2}$ mixed fuzzy topological space.

Let $x_{\alpha}$ and $y_{\beta}$ be two fuzzy points such that $x \neq y$. Therefore, open fuzzy sets $O_{1}$ and $O_{2}$ exist such that $x_{\alpha} \in O_{1} \subseteq$ $c o\left(y_{\beta}\right), y_{\beta} \in O_{2} \subseteq c o\left(x_{\alpha}\right)$, and $O_{1} \subseteq \operatorname{co}\left(O_{2}\right)$. Therefore, $x_{\alpha} \in O_{1} \subseteq \operatorname{co}\left(y_{\beta}\right)$ and $y_{\beta} \in O_{2} \subseteq \operatorname{co}\left(x_{\alpha}\right)$; as such, $(X$, $\left.\tau_{1}\left(\tau_{2}\right)\right)$ is a $T_{1}$ mixed fuzzy topological space.

Let $\left(X, \tau_{1}\left(\tau_{2}\right)\right)$ be a $T_{1}$ mixed fuzzy topological space.

Let $x_{\alpha}$ and $y_{\beta}$ be two fuzzy points such that $x \neq y$. Therefore, open fuzzy sets $O_{1}$ and $O_{2}$ exist such that $x_{\alpha} \in O_{1} \subseteq$ $\operatorname{co}\left(y_{\beta}\right)$ and $y_{\beta} \in O_{2} \subseteq \operatorname{co}\left(x_{\alpha}\right)$. Consequently, $x_{\alpha} \in O_{1} \subseteq$ $\operatorname{co}\left(y_{\beta}\right)$ or $y_{\beta} \in O_{2} \subseteq \operatorname{co}\left(x_{\alpha}\right)$. Hence, $\left(X, \tau_{1}\left(\tau_{2}\right)\right)$ is a mixed fuzzy $T_{0}$ topological space. 


\section{Conclusion}

In this study, we defined fuzzy- $T_{0}$, fuzzy- $T_{1}$, fuzzy- $T_{2}$ (or Hausdorff), fuzzy regular, and fuzzy normal spaces in mixed fuzzy topological spaces. We established the relationships among fuzzy- $T_{0}$, fuzzy- $T_{1}$, fuzzy- $T_{2}$, fuzzy regular, and fuzzy normal spaces in mixed fuzzy topological spaces. Only a few theorems have been established to investigate separation axioms in mixed fuzzy topological spaces. More properties and theorems pertaining to separation axioms will be established in the near future using these established results in the context of mixed fuzzy topological spaces.

\section{Conflict of Interest}

No potential conflict of interest relevant to this article was reported.

\section{References}

[1] A. Alexiewicz and Z. Semadeni, "A generalization of two norm spaces," Bulletin of the Polish Academy of Sciences Mathematics, vol. 6, pp. 135-139, 1958.

[2] J. B. Cooper, "The strict topology and spaces with mixed topologies," Proceedings of the American Mathematical Society, vol. 30, no. 3, pp. 583-592, 1971. https://doi.org/ 10.1090/S0002-9939-1971-0284789-2

[3] R. C. Buck, "Operator algebras and dual spaces," Proceedings of the American Mathematical Society, vol. 3, no. 5, pp. 681-687, 1952. https://doi.org/10.2307/2032166

[4] A. Wiweger, "Linear spaces with mixed topology," Studia Mathematica, vol. 20, no. 1, pp. 47-68, 1961.

[5] L. A. Zadeh, "Fuzzy sets," Information and Control, vol. 8, no. 3, pp. 338-353, 1965. https://doi.org/10.1016/S00199958(65)90241-X

[6] C. L. Chang, "Fuzzy topological spaces," Journal of Mathematical Analysis and Applications, vol. 24, no. 1, pp. 182190, 1968. https://doi.org/10.1016/0022-247X(68)900577

[7] M. M. Stadler and M. A. de Prada Vicente, "Strong separation and strong countability in fuzzy topological spaces," Fuzzy Sets and Systems, vol. 43, no. 1, pp. 95-116, 1991. https://doi.org/10.1016/0165-0114(91)90024-K
[8] N. R. Das and P. C. Baishya, "Mixed fuzzy topological spaces," Journal of Fuzzy Mathematics, vol. 3, no. 4, pp. 777-784, 1995.

[9] B. C. Tripathy and G. C. Ray, "On Mixed fuzzy topological spaces and countability," Soft Computing, vol. 16, pp. 1691-1695, 2012. https://doi.org/10.1007/s00500-0120853-1

[10] M. H. Ghanim, E. E. Kerre, and A. S. Mashhour, "Separation axioms, subspaces and sums in fuzzy topology," Journal of Mathematical Analysis and Applications, vol. 102, no. 1, pp. 189-202, 1984. https://doi.org/10.1016/0022$247 x(84) 90212-9$

[11] W. F. Al-Omeri, "On mixed b-fuzzy topological spaces," International Journal of Fuzzy Logic and Intelligent Systems, vol. 20, no. 3, pp. 242-246, 2020. https://doi.org/10. 5391/IJFIS.2020.20.3.242

[12] M. E. El-Shafei, M. Abo-Elhamayel, and T. M. Al-Shami, "Partial soft separation axioms and soft compact spaces," Filomat, vol. 32, no. 13, pp. 4755-4771, 2018. https://doi. org/10.2298/FIL1813755E

[13] T. M. Al-Shami and M. E. El-Shafei, "On supra soft topological ordered spaces," Arab Journal of Basic and Applied Sciences, vol. 26, no. 1, pp. 433-445, 2019. https://doi.org/10.1080/25765299.2019.1664101

[14] T. M. Al-Shami, M. E. El-Shafei, and M. Abo-Elhamayel, "On soft topological ordered spaces," Journal of King Saud University-Science, vol. 31, no. 4, pp. 556-566, 2019. https://doi.org/10.1016/j.jksus.2018.06.005

[15] T. M. Al-Shami and M. E. El-Shafei, "Two types of separation axioms on supra soft topological spaces," Demonstratio Mathematica, vol. 52, no. 1, pp. 147-165, 2019. https://doi.org/10.1515/dema-2019-0016

[16] T. M. Al-Shami, "Soft separation axioms and fixed soft points using soft semiopen sets," Journal of Applied Mathematics, vol. 2020, article no. 1746103, 2020. https: //doi.org/10.1155/2020/1746103

[17] T. M. Al-Shami, E. A. Abo-Tabl, and B. A. Asaad, "Weak forms of soft separation axioms and fixed soft points," Fuzzy Information and Engineering, vol. 12, no. 4, pp. 509-528, 2020. https://doi.org/10.1080/16168658.2021. 1924528 
[18] T. M. Al-Shami, "On soft separation axioms and their applications on decision-making problem," Mathematical Problems in Engineering, vol. 2021, article no. 8876978, 2021. https://doi.org/10.1155/2021/8876978

[19] T. M. Al-Shami, "Compactness on soft topological ordered spaces and its application on the information system," Journal of Mathematics, vol. 2021, article no. 6699092, 2021. https://doi.org/10.1155/2021/6699092

[20] T. M. Al-Shami, A. Tercan, and A. Mhemdi, "New soft separation axioms and fixed soft points with respect to total belong and total non-belong relations," Demonstratio Mathematica, vol. 54, no. 1, pp. 196-211, 2021. https: //doi.org/10.1515/dema-2021-0018

[21] T. M. Al-Shami and E. A. Abo-Tabl, "Soft a-separation axioms and a-fixed soft points," AIMS Mathematics, vol. 6, no. 6, pp. 5675-5694, 2021. https://doi.org/10.3934/ math.2021335

[22] M. H. Rashid and D. M. Ali, "Separation axioms in mixed fuzzy topological spaces," Journal of Bangladesh Academy of Sciences, vol. 32, no. 2, pp. 142-150, 2008. https://doi.org/10.3329/jbas.v32i2.2433

[23] P. M. Pu and Y. M. Liu, "Fuzzy topology. I. Neighborhood structure of a fuzzy point and Moore-Smith convergence," Journal of Mathematical Analysis and Applications, vol. 76, no. 2, pp. 571-599, 1980. https://doi.org/10.1016/0022247X(80)90048-7
[24] R. Lowen, "Fuzzy topological spaces and fuzzy compactness," Journal of Mathematical Analysis and Applications, vol. 56, no. 3, pp. 621-633, 1976. https://doi.org/10.1016/ 0022-247X(76)90029-9

[25] D. M. Ali, "A note on fuzzy regularity concepts," Fuzzy Sets and Systems, vol. 35, no. 1, pp. 101-104, 1990. https: //doi.org/10.1016/0165-0114(90)90022-X

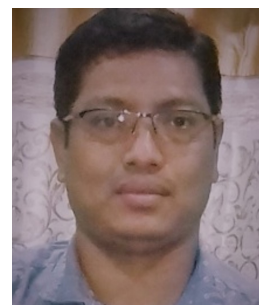

Gautam Chandra Ray received a Ph.D. degree in Mathematics from Gauhati University, Assam, India. He is an assistant professor in the Department of Mathematics in CIT Kokrajhar, Assam, India. His research interests include neutrosophic sets and logic, fuzzy sets and systems, topology, and graph theory.

E-mail: gautomofcit@gmail.com

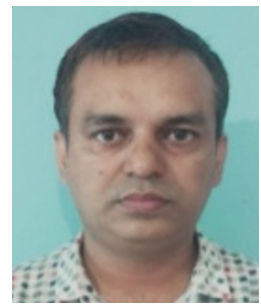

Hari Prasad Chetri received his M.Sc. degree in mathematics from Gauhati University, India. He is a research scholar in the Department of Mathematics of the Central Institute of Technology, Kokrajhar, India. His research interests include general topology, fuzzy topology, and algebra.

E-mail: hariprasadchetri1234@gmail.com 